\title{
A donor-specific epigenetic classifier for acute graft-versus-host disease severity in hematopoietic stem cell transplantation
}

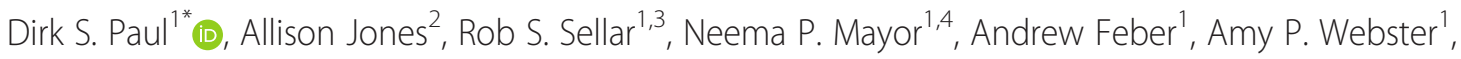
Neuza Afonso ${ }^{5}$, Ruhena Sergeant ${ }^{6}$, Richard M. Szydlo ${ }^{7,8}$, Jane F. Apperley ${ }^{7,8}$, Martin Widschwendter ${ }^{2}$, Stephen Mackinnon ${ }^{1,5}$, Steven G. E. Marsh ${ }^{1,4}$, J. Alejandro Madrigal ${ }^{1,4}$, Vardhman K. Rakyan ${ }^{9^{*}}$, Karl S. Peggs ${ }^{1,3^{*}}$ and Stephan Beck ${ }^{1 *}$

\begin{abstract}
Background: Allogeneic hematopoietic stem cell transplantation (HSCT) is a curative treatment for many hematological conditions. Acute graft-versus-host disease (aGVHD) is a prevalent immune-mediated complication following HSCT. Current diagnostic biomarkers that correlate with aGVHD severity, progression, and therapy response in graft recipients are insufficient. Here, we investigated whether epigenetic marks measured in peripheral blood of healthy graft donors stratify aGVHD severity in human leukocyte antigen (HLA)-matched sibling recipients prior to T cell-depleted HSCT.
\end{abstract}

Methods: We measured DNA methylation levels genome-wide at single-nucleotide resolution in peripheral blood of $85 \mathrm{HSCT}$ donors, matched to recipients with various transplant outcomes, with Illumina Infinium HumanMethylation450 BeadChips.

Results: Using genome-wide DNA methylation profiling, we showed that epigenetic signatures underlying aGVHD severity in recipients correspond to immune pathways relevant to aGVHD etiology. We discovered 31 DNA methylation marks in donors that associated with aGVHD severity status in recipients, and demonstrated strong predictive performance of these markers in internal cross-validation experiments ( $\mathrm{UUC}=0.98,95 \% \mathrm{Cl}=0.96-0.99$ ). We replicated the top-ranked CpG classifier using an alternative, clinical DNA methylation assay $(P=0.039)$. In an independent cohort of $32 \mathrm{HSCT}$ donors, we demonstrated the utility of the epigenetic classifier in the context of a T cell-replete conditioning regimen $(P=0.050)$.

Conclusions: Our findings suggest that epigenetic typing of HSCT donors in a clinical setting may be used in conjunction with HLA genotyping to inform both donor selection and transplantation strategy, with the ultimate aim of improving patient outcome.

\section{Background}

Hematopoietic stem cell transplantation (HSCT) is a curative therapy for a wide range of hematological disorders and malignancies. Severe immune reactions, in particular graft-versus-host disease (GVHD), can decrease HSCT efficiency and survival in patients [1]. Immunosuppressive agents that counteract such events confer

\footnotetext{
*Correspondence: d.paul@ucl.ac.uk; v.rakyan@qmul.ac.uk; karl.peggs@ucl.ac. uk; s.beck@ucl.ac.uk

'UCL Cancer Institute, University College London, London, UK

${ }^{9}$ Blizard Institute, Barts and The London School of Medicine and Dentistry,

Queen Mary University of London, London, UK

Full list of author information is available at the end of the article
}

further complications, such as opportunistic infections and cancer recurrence [2].

Acute GVHD (aGVHD) has classically been described to develop within 100 days after HSCT, but can sometimes occur at later time points. In aGVHD, alloreactive donor $\mathrm{T}$ cells respond to antigens in the host tissues and damage recipient epithelial cells in skin, liver, and gastrointestinal tract [3]. T cell depletion of donor graft provides an efficient strategy of reducing the incidence of aGVHD, but can delay immune reconstitution and abrogate beneficial graft-versus-tumor effects [4]. Without $\mathrm{T}$ cell depletion, aGVHD affects $20-40 \%$ of graft recipients when the donor and recipient are related, and 
40-70\% when they are not related [5]. The incidence depends on a number of factors, including relatedness and degree of human leukocyte antigen (HLA) disparity, as well as differences in sex, age, and cytomegalovirus serostatus, between donor and recipient.

Promising novel therapeutic approaches to prevent or treat GVHD are being developed, including monoclonal antibodies targeting inflammatory cytokines and smallmolecule inhibitors that alter immune cell trafficking (reviewed in [1]). In parallel, biomarkers that inform the risk of development and severity of GVHD are of substantial clinical importance. Several studies have measured plasma concentration levels of a number of different proteins, such as IL2RA and ST2, demonstrating correlation with responsiveness to treatment $[6,7]$. Importantly, all biomarkers thus far characterized are applied in recipients after HSCT. Biomarkers that guide transplantation strategy have not yet been identified, but could provide a valuable approach to improving patient outcome following HSCT.

Epigenetic factors, such as DNA methylation and posttranscriptional modification of histones, play a critical role in regulating gene transcriptional programs that dictate immune cell fate and function [8]. Epigenetic mechanisms in circulating immune cells are sensitive to environmental factors and may contribute to disease development and progression, alongside genetic predisposition. For example, epigenetic mechanisms have been uncovered for distinct $\mathrm{T}$ cell differentiation pathways [9-11], and DNA methylation patterns have been associated with inflammatory and autoimmune disease susceptibility, including type 1 diabetes [12], systemic lupus erythematosus [13], and rheumatoid arthritis [14].

However, thus far little attention has been paid to the possible impact of epigenetic factors on HSCT outcomes. To this end, Rodriguez et al. examined DNA methylation differences in peripheral blood between donors and recipients ( $\mathrm{n}=47$ pairs), both pre- and post-HSCT [15]. Global DNA methylation levels were estimated at $\mathrm{CpG}$ sites at repetitive DNA elements using a pyrosequencing-based assay. The results suggest that recipients maintain the donor's global methylation levels after HSCT. DNA methylation levels were further measured at promoters of genes with functions relevant to immune responses in HSCT. In this analysis, the authors identified subtle DNA methylation changes at the IFNG, FASLG, and IL10 gene promoters between recipients developing either no or mild, and severe aGVHD one month after HSCT.

Differential DNA methylation analyses between HSCT donors and recipients are impeded by several factors. First, recipients that are appropriate for HSCT suffer from a wide range of hematological malignancies. Epigenetic dysregulation in cancer etiology is well-described
[16]; therefore, the meaningful comparison of DNA methylation patterns with regards to HSCT between healthy donors and patients is unattainable. Second, blood cells isolated from post-HSCT recipients may originate either from the remaining hematopoietic repertoire or the donor graft (that is, 'mixed chimerism'), complicating the interpretation of the derived epigenetic signature.

In the present study, we investigated whether distinct epigenetic marks in peripheral blood of healthy graft donors delineate aGVHD severity in HLA-matched sibling recipients prior to HSCT. We measured DNA methylation levels genome-wide at 414,827 CpG sites at singlenucleotide resolution in peripheral blood of 85 HSCT donors, matched to recipients with various transplant outcomes. We defined a DNA methylation signature that stratifies graft donors with respect to aGVHD severity diagnosed in recipients, and replicated the signature with an alternative DNA methylation assay used in a routine clinical diagnostics environment. Here, we introduce the approach of epigenetic typing of HSCT donors to be used in conjunction with HLA genotyping to inform both donor selection and transplantation strategy.

\section{Methods}

\section{Ethics}

The research conformed to the Declaration of Helsinki and to local regulatory legislation. All HSCT donors and patients gave written informed consent according to local institutional guidance and JACIE (Joint Accreditation Committee of the International Society for Cellular Therapy and the European Group for Blood and Marrow Transplantation) standards for the analyses performed and publication of these data. The study was approved by the UCL Research Ethics Committee (Project ID 7759/001).

\section{Experimental design}

The discovery cohort consisted of 85 HLA-identical sibling pairs who underwent reduced-intensity allogeneic HSCT between June 2000 and November 2012 at either the University College London Hospital or Royal Free Hospital (London, UK). Sibling pairs were 10/10 HLA allele-matched (that is, for $H L A-A,-B,-C,-D R B 1$, and $-D Q B 1)$. Donors provided peripheral blood stem cells mobilized by granulocyte-colony stimulating factor (G-CSF). All recipients received uniform conditioning with fludarabine, melphalan, and alemtuzumab [17]. Acute and chronic GVHD were assessed and graded according to published criteria [18]. Cyclosporine A was administered for GVHD prophylaxis. In the absence of GVHD, immunosuppression was decreased from three months after HSCT. The 85 graft donors were matched with recipients of various transplant outcomes: 'severe' 
aGVHD (grades III + IV; $\mathrm{n}=9$ ), ' mild' aGVHD (grades I + II; $\mathrm{n}=37)$, and no aGVHD $(\mathrm{n}=39)$. To obtain more equally powered sample groups, we enriched for severe transplant outcomes.

To assess the initial findings with regards to transplant conditioning regimen, we identified a validation cohort consisting of 32 HLA-identical sibling pairs undergoing T cell-replete HSCT between September 2000 and April 2012 at Hammersmith Hospital (London, UK). One of three regimens was used: (1) fludarabine alone; (2) fludarabine, rituximab, and cyclophosphamide; or (3) lomustine, cytarabine, cyclophosphamide, and etoposide. Patients received cyclosporine $\mathrm{A}$ and methotrexate as prophylaxis against GVHD. The 32 graft donors were matched with recipients of the following transplant outcomes: severe aGVHD $(n=9)$, mild aGVHD $(n=8)$, and no aGVHD $(\mathrm{n}=15)$.

\section{DNA extraction}

DNA was extracted from peripheral blood using a QIAamp DNA Blood BioRobot MDx Kit (QIAGEN) following the manufacturer's instructions. The DNA concentration was assessed using a Qubit dsDNA BR Assay Kit (Invitrogen).

\section{Illumina Infinium HumanMethylation450 assay}

Genomic DNA was bisulfite-converted using an EZ-96 DNA Methylation MagPrep Kit (Zymo Research) according to the manufacturer's instructions. We applied $500 \mathrm{ng}$ or $250 \mathrm{ng}$ of genomic DNA to bisulfite treatment, and eluted purified, bisulfite-converted DNA in $20 \mu \mathrm{L}$ or $11 \mu \mathrm{L}$ of M-Elution Buffer (Zymo Research), respectively. DNA methylation levels were measured using Infinium HumanMethylation450 assays (Illumina) following the manufacturer's protocol. In brief, $4 \mu \mathrm{L}$ of bisulfite-converted DNA was isothermally amplified, enzymatically fragmented, and precipitated. Next, precipitated DNA was resuspended in hybridization buffer and dispensed onto Infinium HumanMethylation450 BeadChips (Illumina). To limit batch effects, samples were randomly distributed across slides and arrays. The hybridization was performed at $48{ }^{\circ} \mathrm{C}$ for $20 \mathrm{~h}$ using a Hybridization Oven (Illumina). After hybridization, BeadChips were washed and processed through a singlenucleotide extension followed by immunohistochemistry staining using a Freedom EVO Robot (Tecan). Finally, the BeadChips were imaged using an iScan Microarray Scanner (Illumina).

\section{Illumina Infinium HumanMethylation450 data preprocessing}

The DNA methylation fraction at a specific $\mathrm{CpG}$ site was calculated as $\beta=M /(M+U+100)$, for which $M$ and $\mathrm{U}$ denote methylated and unmethylated fluorescent signal intensities, respectively. The $\beta$-value statistic ranges from absent $(\beta=0)$ to complete DNA methylation $(\beta=1)$ at a particular CpG site. We normalized the $450 \mathrm{~K}$ array data using Functional Normalization (FunNorm), a novel between-array normalization method, which is based on quantile normalization and uses control probes to act as surrogates for unwanted variation [19, 20]. In addition, the method entails background correction and dye-bias normalization using NOOB [21]. Next, we filtered: (1) probes with median detection $P$ value $\geq 0.01$ in one or more samples; (2) probes with bead count of less than three in at least $5 \%$ of samples; (3) probes mapping to sex chromosomes; (4) non-CG probes; (5) probes mapping to ambiguous genomic locations [22]; and (6) probes harboring SNPs at the probed CG irrespective of allele frequency in the Asian, American, African, and European populations based on the 1000 Genomes Project (Release v3, 2011-05-21). All 450K array data preprocessing steps were carried out using the $R$ package minfi [20]. Finally, we adjusted for batch effects (Sentrix ID) using an empirical Bayesian framework [23], as implemented in the ComBat function of the $\mathrm{R}$ package SVA [24]. The 450K array data generated as part of this study have been submitted to the European Genomephenome Archive (https://www.ebi.ac.uk/ega/) with accession number EGAS00001001287.

\section{Estimation of differential leukocyte counts}

We estimated differential leukocyte counts for each individual using an algorithm that is based on regression calibration [25], and implemented in the $R$ package minfi [20]. In brief, for each sample the relative proportions of principal leukocyte cell types was inferred using DNA methylation signatures of an external validation set consisting of purified leukocytes, specifically $\mathrm{CD}^{+} \mathrm{CD}^{+}$and $\mathrm{CD}^{+} \mathrm{CD}^{+} \mathrm{T}$ lymphocytes, $\mathrm{CD} 19^{+} \mathrm{B}$ lymphocytes, $\mathrm{CD}^{+} 6^{+}$natural killer cells, $\mathrm{CD} 14^{+}$monocytes, and $\mathrm{CD} 15^{+}$granulocytes.

\section{Identification of differentially methylated regions (DMRs) and positions (DMPs)}

We identified DMRs associated with aGVHD severity using Probe Lasso v6.1 [26]. We applied the following parameters: lassoStyle $=\max$, lassoRadius $=2000$, minSigProbesLasso $=2, \operatorname{minDmrSep}=1000, \operatorname{minDmrSize}=$ 0 , and adjPVal $=0.1 . P$ values of DMRs were corrected for multiple testing with the false discovery rate (FDR) method. To identify DMPs, we fitted a linear regression model predicting DNA methylation state at each CpG site as a function of aGVHD severity (severe $=1$ vs. no/ mild aGVHD $=0$ ), adjusted for sex, age at graft donation, and estimated differential cell counts $(\mathrm{CD} 8 \mathrm{~T}+\mathrm{CD} 4 \mathrm{~T}+$ Bcell + NK + Mono + Gran). The DMP analysis was performed using the $\mathrm{R}$ package limma [27]. The approach 
uses an empirical Bayes method to moderate the standard errors of the estimated log-fold changes. $P$ values of identified DMPs were corrected for multiple testing using the Bonferroni method.

\section{Annotation of DMRs using the Genomic Regions Enrichment of Annotations Tool (GREAT)}

We analyzed the ontology of genes flanking the identified DMRs with GREAT v3.0.0 [28], using the standard parameters: association rule $=$ basal + extension (constitutive $5 \mathrm{~kb}$ upstream, $1 \mathrm{~kb}$ downstream, up to $1 \mathrm{Mb}$ extension); curated regulatory domains = included; background $=$ whole genome.

\section{Assessment of epigenetic classifier performance with leave-one-out cross-validation (LOOCV)}

To assess the performance of the epigenetic classifier, we used the preprocessed $450 \mathrm{~K}$ array data set consisting of 85 donors. In each iteration of the LOOCV, one sample was left out and DMPs were identified using the remaining dataset ( $\mathrm{n}=85-1$ donor samples). We used the same linear regression model, covariates, and significance thresholds for identifying DMPs as described above. Significant DMPs were ranked according to their $P$ values. Then, a nearest shrunken centroid classifier was trained on the identified DMPs, as implemented in the $\mathrm{R}$ package pamr $[29,30]$. The number of crossvalidation folds was specified to the smallest class size, and a (random) balanced cross-validation was used (default parameters). The threshold for centroid shrinkage was set to one. The resulting centroid classifiers were used to predict aGVHD severity status on the omitted sample. Finally, classifier performance was evaluated using receiver operating characteristic (ROC) and area under the curve (AUC) measures, as implemented in the $\mathrm{R}$ package pROC [31].

\section{Measurement of relative DNA methylation levels using MethyLight}

Genomic DNA was bisulfite-converted using an EZ DNA Methylation-Gold Kit (Zymo Research) according to the manufacturer's instructions. PCR primers and probes for MethyLight analyses were designed specifically for bisulfite-converted DNA ( $5^{\prime}$ to $3^{\prime}$ plus strand) using ABI Primer Express v3. All oligonucleotides were synthesized by Metabion. Details with regards to PCR primers and probes used in this study are provided in Additional file 1 . The reaction for the $\mathrm{CpG}$ of interest was assayed alongside a reference, the collagen 2A1 gene (COL2A1), to normalize for input DNA. Specificity of the reactions for methylated DNA was confirmed using M.SssI-treated human peripheral blood lymphocyte DNA (fully methylated positive control), whole-genome amplified DNA (unmethylated negative control), and a non-template control. The efficiencies of primers were assessed using a five-log serial dilution of M.SssI-treated human genomic standard. In addition, an agarose gel was run to ensure a single and appropriately sized PCR product. The fraction of fully methylated molecules at a specific locus was represented as percentage of methylated reference (PMR). First, all $\mathrm{C}_{\mathrm{t}}$-values were interpolated from the standard curve based on a four-fold dilution of M.SssI-treated DNA. Then, we calculated PMR values by dividing the target $\mathrm{CpG}$ /reference $\mathrm{C}_{\mathrm{t}}$-ratio of a sample by the $\mathrm{CpG} /$ reference $\mathrm{C}_{\mathrm{t}}$-ratio of the M.SssI-treated DNA, multiplied by 100. All MethyLight reactions were performed on a 6FLX Real-Time PCR System (Life Technologies). DNA methylation thresholds with the maximal specificity and sensitivity were determined at the coordinates closest to the top-left part of the ROC curves (best.method = closest.topleft), as implemented in the R package pROC [31].

\section{Software for statistical analyses}

All statistical analyses described in this study were performed using R v3.1.1 and Bioconductor v3.0.

\section{Results}

Characterization of distinct genome-wide DNA methylation signatures in HSCT donors

We investigated a total of 85 HLA-identical HSCT donor-recipient sibling pairs. We focused on sibling pairs to minimize the contribution from genetic factors in our analyses. All patients undergoing HSCT received reduced-intensity (non-myeloablative) $\mathrm{T}$ cell-depleted conditioning using in vivo alemtuzumab. The $\mathrm{T}$ celldepleted platform was chosen in the first instance in order to try to identify major drivers of aGVHD in the context of a platform with a relatively low incidence of GVHD. Sample selection was enriched for severe transplant outcomes to balance sample groups, that is, 'severe' aGVHD (grades III + IV; n=9), 'mild' aGVHD (grades I + II; $\mathrm{n}=37$ ), and no aGVHD (grade 0; $\mathrm{n}=39$ ). Details about sample selection are described in the Methods section, and demographics of HSCT donors and recipients are provided in Table 1 . An overview of the study design is illustrated in Fig. 1.

We measured genome-wide DNA methylation levels in peripheral blood of HSCT donors using Illumina Infinium HumanMethylation450 BeadChips ('450K arrays'). The two-color array allows the assessment of DNA methylation status at over 485,000 CpG sites at singlenucleotide resolution. The assay covers $99 \%$ of RefSeq genes with an average of $17 \mathrm{CpG}$ sites per gene region, and $96 \%$ of CpG islands [32]. Array data preprocessing was performed using established analytical methods (Methods). Array probes were filtered with stringent quality criteria, leaving a total of $414,827 \mathrm{CpG}$ sites for 
Table 1 Demographics of HSCT donors and recipients in the discovery and validation cohorts

$\frac{\frac{\text { Discovery cohort }}{(n=85 \text { pairs })} \frac{\text { Validation cohort }}{(n=32 \text { pairs })}}{\text { Donors Recipients }}$

Sex

\section{Female}

Male

Age at transplant (years)

Median

Range

CMV serostatus

Positive

Negative

NA

Max. acute GVHD

Absent

Grade I ('mild')

Grade II ('mild')

Grade III ('severe')

Grade IV ('severe')

Max. chronic GVHD

Absent
Limited
Extensive
NA

Diagnosis

Acute lymphoblastic leukemia

Acute myeloid leukemia

Chronic granulomatous

disease

Chronic lymphocytic

leukemia (CLL)

Chronic myeloid leukemia (CML)

Chronic myelomonocytic leukemia

Hodgkin lymphoma

Myelodysplasia (MDS)

MDS/CML/CLL

Myelofibrosis

Myeloproliferative syndromes/MDS

Myeloma

Non-Hodgkin lymphoma

Other

NA
Donors Recipients Donors Recipients

$\begin{array}{llll}41 & 27 & 14 & 7 \\ 44 & 58 & 18 & 25\end{array}$

$\begin{array}{llll}48 & 52 & 53 & 55\end{array}$

$14-72 \quad 21-66 \quad 22-71 \quad 23-64$

$\begin{array}{llll}38 & 43 & 14 & 11\end{array}$

$\begin{array}{llll}31 & 32 & 16 & 21\end{array}$

$\begin{array}{llll}16 & 10 & 2 & 0\end{array}$

39

- $\quad 39$

- 24

- 13

- 8

$\begin{array}{ll}- & 1\end{array}$

- 54

- $\quad 21$

- 9

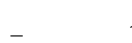

$-$

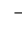

$\begin{array}{llll}- & 11 \quad- & 0\end{array}$

$-$

$\begin{array}{llll}- & 1 & - & 0 \\ - & 9 & - & 4 \\ - & 2 & - & 0 \\ - & 1 & - & 0 \\ - & 0 & - & 2 \\ - & 0 & - & 2 \\ - & 3 & - & 0 \\ - & 37 & - & 8 \\ - & 0 & - & 4 \\ - & 2 & - & 0\end{array}$

Table 1 Demographics of HSCT donors and recipients in the discovery and validation cohorts (Continued)

\begin{tabular}{lcc}
\hline & Sibling pairs & Sibling pairs \\
Sex match (donor/recipient) & 30 & 14 \\
Male/male & 13 & 3 \\
Female/female & 14 & 4 \\
Male/female & 28 & 11 \\
Female/male & & \\
CMV match (donor/recipient) & 18 & 13 \\
Negative/negative & 25 & 8 \\
Positive/positive & 13 & 3 \\
Negative/positive & 13 & 6 \\
Positive/negative & 16 & 2 \\
NA
\end{tabular}

Detailed information regarding criteria for study inclusion is provided in the Methods section. Note that the diagnosis 'Non-Hodgkin lymphoma' includes various disease subtypes.

CMV cytomegalovirus, NA data not available

subsequent statistical analyses. A summary of the quality assessment of the $450 \mathrm{~K}$ array data is shown in Additional file 2.

We performed multidimensional scaling (MDS) based on all measured CpG sites to assess the degree of similarity of individual HSCT donors. HSCT donors matched to healthy recipients and those matched to recipients diagnosed with mild aGVHD could not be discriminated using MDS (Additional file 2). Consequently, these two sample groups were combined for subsequent analyses. The analytical approach identified a DNA methylation signature that stratifies donors paired with recipients with severe aGVHD (Additional file 2).

To characterize the DNA methylation signatures underlying aGVHD severity, we identified DMRs between donors paired with no/mild aGVHD and severe aGVHD. DMRs have been shown to more likely locate near differentially expressed genes compared to differentially methylated single CpG sites [20]. DMRs were identified using the Probe Lasso algorithm [26], which applies a dynamic window based on probe annotation and density to record neighboring significant $\mathrm{CpG}$ sites and determine discrete DMR boundaries. A total of 453 DMRs at an FDR of less than $10 \%$ were discovered. We annotated the genes flanking these DMRs using the Genomic Regions Enrichment of Annotations Tool (GREAT) [28], and observed enrichment in the ontology terms 'MHC class II receptor activity' (GO Molecular Function; $P=3.53 \times 10^{-5}, \quad$ FDR-corrected binomial test), 'MHC class II protein complex' (GO Cellular Component; $P=4.46 \times 10^{-6}$ ), 'antigen processing and presentation' (MSigDB Gene Sets Canonical Pathway; $\left.P=2.08 \times 10^{-6}\right)$, 'MHC classes I/II-like antigen recognition protein' (InterPro; $P=1.97 \times 10^{-4}$ ), among other 


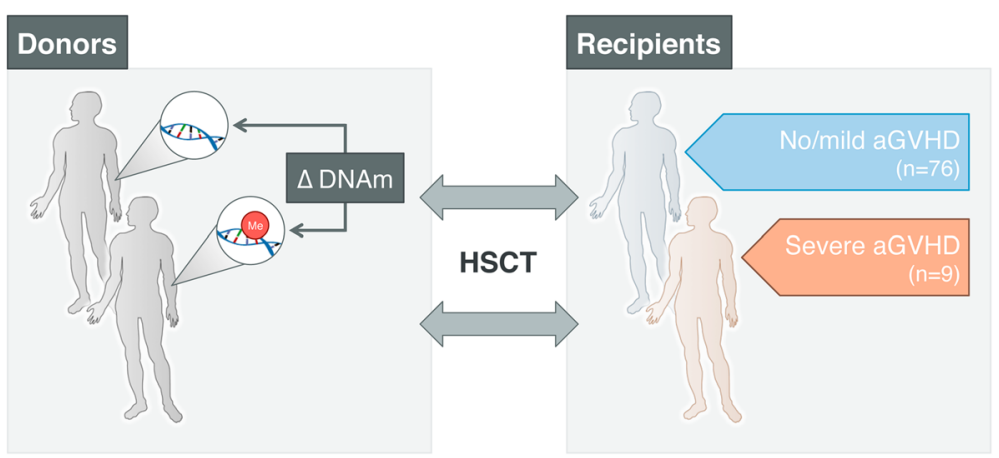

Fig. 1 Overview of the study design. We aimed to identify specific epigenetic marks in peripheral blood of healthy graft donors that delineate aGVHD severity in HLA-matched sibling recipients prior to HSCT. At the discovery stage, we assessed genome-wide DNA methylation levels in peripheral blood of $85 \mathrm{HSCT}$ donors, matched to recipients with various transplant outcomes, that is, 'severe' aGVHD (grades III + IV; $n=9)$ and 'no/mild' aGVHD (grades $0,1+\| ; n=76$ ). HSCT recipients received reduced-intensity (non-myeloablative) $T$ cell-depleted conditioning using in vivo alemtuzumab. At the replication stage, we used a semi-quantitative DNA methylation assay, MethyLight, which can be easily used in a clinical setting. We validated the top-ranked differentially methylated positions associated with aGVHD severity status in donors in the context of both T cell-depleted and T cell-replete conditioning regimens for HSCT

relevant terms (Additional file 3). Taken together, these results indicate that healthy HSCT donors whose recipients develop severe aGVHD exhibit a specific DNA methylation signature, which correlates with known molecular processes relevant to GVHD pathobiology.

\section{Identification of differentially methylated positions associated with aGVHD severity}

Next, we determined DMPs in HSCT donors associated with aGVHD severity in recipients that may be exploited as biomarkers for clinical diagnostics. We used a linear regression model predicting DNA methylation state at each CpG site as a function of aGVHD severity status, that is, severe vs. no/mild aGVHD diagnosed in matched graft recipients. In the regression model, we adjusted for sex, age at graft donation, and estimated relative proportions of major leukocyte cell types (Methods). We identified 31 DMPs that achieved a $P$ value $<0.05$ after Bonferroni correction. To ascertain DMPs of potential biological significance, as well as permit validation using a semi-quantitative DNA methylation assay applicable for routine clinical testing, we only considered DMPs with a DNA methylation difference of at least $\pm 5 \%$ (Table 2). Strikingly, four top-ranked DMPs (that is, cg20475486, cg10399005, cg07280807, and cg09284655) form a DMR with consistent DNA hypomethylation in donors matched to recipients with severe aGVHD compared to donors paired with recipients with no/mild aGVHD (Fig. 2a). This locus was also identified as one of the top-ranking DMRs using the Probe Lasso algorithm $\left(P=4.55 \times 10^{-31}\right.$; rank $\left.=2\right)$.

To estimate the epigenetic classifier performance, we used leave-one-out cross-validation (LOOCV). In brief, one donor sample was left out in each iteration of the LOOCV, and DMPs were identified on the remaining sample cohort as described above. Then, a nearest shrunken centroid classifier was trained on the identified DMPs (Methods). The resulting classifiers were used to predict aGVHD severity status on the sample that was left out. Centroid classifier performance was evaluated by means of ROC curves and summarized by AUC values. Over the 85 iterations, the mean AUC was 0.98 (95\% confidence interval $=0.96-0.99$; Fig. $2 b$ ). Importantly, all four DMPs contained within the DMR (Fig. 2a) were selected in over $90 \%$ of iterations of the LOOCV classifier (Table 2). Our data indicate discovery of discrete DMPs that discriminate aGVHD severity status, and demonstrate strong predictive performance in internal cross-validation experiments.

\section{Replication of top-ranked differentially methylated posi-} tions using a clinical biomarker assay

Following the discovery of DMPs using $450 \mathrm{~K}$ arrays, we aimed to replicate the top-ranked CpG sites using a semiquantitative DNA methylation assay, MethyLight. This well-established assay uses PCR amplification of bisulfiteconverted DNA in combination with fluorescently-labeled probes that hybridize specifically to a fully methylated DNA sequence [33]. The resulting data is presented as a percentage relative to an M.SssI-treated, fully methylated DNA reference sample (PMR). While the quantitative accuracy is lower compared to Illumina Infinium and next-generation DNA sequencing-based assays, MethyLight can be readily translated into a clinical setting at relatively low cost $[34,35]$.

We focused our replication efforts on the highly discriminative DMPs located at the DMR on chromosome 14q24.2 (Table 2; Fig. 2a). We designed MethyLight reactions targeting three DMPs, cg20475486, cg10399005, and cg07280807. Through thorough assessment of the 
Table 2 Significant DMPs associated with aGVHD severity status

\begin{tabular}{|c|c|c|c|c|c|c|c|c|c|}
\hline Rank & DMP & Adj. $P$ value & $\Delta \beta$-value & Chr. & Position & Gene & Feature & CpG context & LOOCV \\
\hline 1 & cg20475486 & $1.38 \times 10^{-3}$ & -0.180 & 14 & $70,317,075$ & - & IGR & Island & 85 \\
\hline 2 & cg10399005 & $1.62 \times 10^{-4}$ & -0.162 & 14 & $70,316,898$ & - & IGR & Island & 85 \\
\hline 3 & cg16925210 & $1.60 \times 10^{-5}$ & 0.130 & 2 & $216,946,718$ & PECR & TSS200 & Island & 85 \\
\hline 4 & cg00762468 & $7.61 \times 10^{-6}$ & 0.066 & 16 & 103,568 & POLR3K & 1stExon & Island & 85 \\
\hline 5 & cg26758857 & $1.07 \times 10^{-7}$ & -0.062 & 22 & $36,649,135$ & APOL 1 & $5^{\prime} U T R$ & - & 85 \\
\hline 6 & cg21752471 & $8.92 \times 10^{-4}$ & -0.061 & 5 & $133,861,794$ & PHF15 & TSS200 & Island & 85 \\
\hline 7 & cg21459486 & $5.14 \times 10^{-4}$ & -0.060 & 13 & $112,885,426$ & - & IGR & - & 85 \\
\hline 8 & $\operatorname{cg} 16495818$ & $2.05 \times 10^{-5}$ & -0.055 & 11 & $73,306,367$ & FAM168A & $5^{\prime} U T R$ & Shelf & 85 \\
\hline 9 & cg16276523 & $5.29 \times 10^{-4}$ & -0.094 & 1 & $161,049,579$ & PVRL4 & Body & Island & 84 \\
\hline 10 & cg19558933 & $5.11 \times 10^{-3}$ & -0.129 & 11 & $134,632,242$ & - & IGR & Island & 82 \\
\hline 11 & cg000877067 & $9.63 \times 10^{-3}$ & 0.071 & 5 & $5,265,509$ & ADAMTS16 & Body & - & 82 \\
\hline 12 & cg14099595 & $1.62 \times 10^{-2}$ & 0.067 & 5 & $145,717,283$ & POU4F3 & TSS1500 & Shore & 82 \\
\hline 13 & cg07464977 & $6.69 \times 10^{-3}$ & -0.062 & 5 & $2,097,136$ & - & IGR & - & 82 \\
\hline 14 & cg11364888 & $8.50 \times 10^{-3}$ & -0.055 & 12 & $116,920,304$ & - & IGR & - & 82 \\
\hline 15 & cg24599107 & $9.57 \times 10^{-3}$ & -0.073 & 1 & $161,049,443$ & PVRL4 & Body & Island & 81 \\
\hline 16 & cg07280807 & $8.94 \times 10^{-3}$ & -0.151 & 14 & $70,317,239$ & - & IGR & Island & 80 \\
\hline 17 & cg14546466 & $2.49 \times 10^{-2}$ & -0.077 & 2 & $11,796,974$ & - & IGR & Island & 80 \\
\hline 18 & cg03481039 & $8.60 \times 10^{-3}$ & -0.065 & 11 & $116,662,012$ & APOA5 & Body & Shore & 80 \\
\hline 19 & cg03896685 & $2.62 \times 10^{-2}$ & -0.062 & 7 & $65,512,561$ & - & IGR & Shelf & 80 \\
\hline 20 & cg00245850 & $1.78 \times 10^{-2}$ & 0.060 & 8 & $143,925,513$ & GML & Body & - & 80 \\
\hline 21 & cg09284655 & $1.17 \times 10^{-2}$ & -0.152 & 14 & $70,317,228$ & - & IGR & Island & 79 \\
\hline 22 & cg10287485 & $2.20 \times 10^{-2}$ & 0.090 & 11 & $69,473,145$ & - & IGR & Shelf & 79 \\
\hline 23 & cg19704238 & $1.55 \times 10^{-2}$ & 0.058 & 10 & $85,900,022$ & GHITM & $5^{\prime} U T R$ & Shore & 79 \\
\hline 24 & cg09282654 & $3.33 \times 10^{-2}$ & 0.070 & 7 & $123,563,992$ & SPAM1 & TSS1500 & - & 75 \\
\hline 25 & cg16701890 & $3.57 \times 10^{-2}$ & 0.064 & 2 & $119,906,008$ & - & IGR & - & 73 \\
\hline 26 & cg04664342 & $3.80 \times 10^{-2}$ & -0.107 & 17 & $4,685,905$ & TM4SF5 & Body & Island & 65 \\
\hline 27 & cg20307347 & $4.06 \times 10^{-2}$ & -0.119 & 2 & $231,734,563$ & ITM2C & Body & Shelf & 58 \\
\hline 28 & cg19311448 & $4.17 \times 10^{-2}$ & -0.075 & 2 & $11,797,017$ & - & IGR & Island & 45 \\
\hline 29 & cg09852744 & $4.55 \times 10^{-2}$ & 0.058 & 6 & $158,732,256$ & TULP4 & TSS1500 & - & 39 \\
\hline 30 & cg16398628 & $4.46 \times 10^{-2}$ & 0.090 & 10 & $91,011,689$ & LIPA & TSS200 & Island & 32 \\
\hline 31 & cg20585841 & $4.57 \times 10^{-2}$ & -0.164 & 8 & $102,729,926$ & NCALD & Body & - & 21 \\
\hline
\end{tabular}

DMPs achieving a $P$ value $<0.05$ after Bonferroni correction and a DNA methylation difference of at least $\pm 5 \%$ are reported. DMPs are ranked according to their recurrence in the LOOCV, for which a total of 85 iterations were performed, and their absolute DNA methylation difference. DNA methylation differences were calculated as follows: mean $\beta$-values of HSCT donors matched to recipients with severe aGVHD - mean $\beta$-values of HSCT donors matched to recipients with no/ mild aGVHD. The four highlighted DMPs on chromosome $14 q 24.2$ form a region with consistent DNA hypomethylation in graft donors matched to recipients with severe aGVHD compared to donors paired with recipients with no/mild aGVHD (Fig. 2a). Chromosomal positions are reported in genome build $=$ hg19

performance characteristics of the individual reactions (Methods), we identified cg20475486 with the highest PCR efficiency. Consequently, we measured relative DNA methylation levels at cg20475486 in 63 of the previous 85 HSCT donor samples, for which sufficient material was available. We replicated the observed DNA hypomethylation phenotype in donors paired with recipients diagnosed with severe aGVHD $(P=0.039$, Wilcoxon rank-sum test; Fig. 3a). At a DNA methylation threshold with the maximal specificity and sensitivity, the AUC was 0.74 (Fig. 3b). Together, our results suggest technically robust identification of DMPs associated with aGVHD severity using both Infinium and MethyLight assays.

\section{Validation of epigenetic classifiers in donors in the context of T cell-replete HSCT}

The discovery and replication of DMPs associated with aGVHD severity was carried out in donors matched to recipients that were subjected to $\mathrm{T}$ cell depletion as part of their transplant conditioning regimen. We next explored whether the identified epigenetic classifier could 

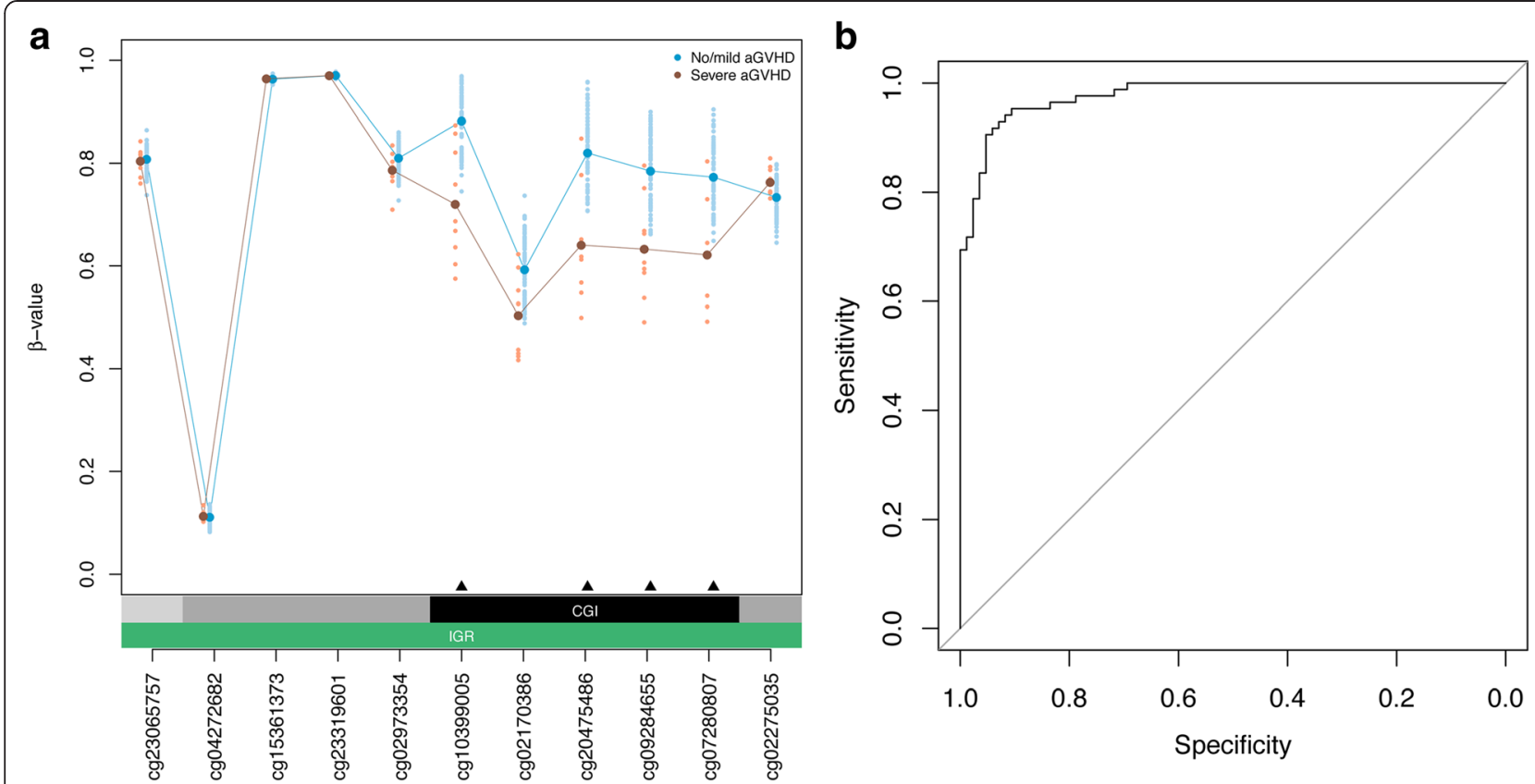

Fig. 2 Identification of DMPs associated with aGVHD severity. a Genomic locus on chromosome 14q24.2 harboring four top-ranked DMPs associated with aGVHD severity. A DNA hypomethylation phenotype was observed in HSCT donors matched to recipients with severe aGVHD (red points) compared to donors paired with recipients with no/mild aGVHD (blue points). Lines represent the means of the measured DNA methylation levels ( $\beta$-values) across HSCT donors. Statistically significant DMPs are indicated with a black triangle. Annotation of all significant DMPs is provided in Table 2. b ROC measures evaluating the epigenetic classifier performance. LOOCV was used to assess the classifier performance (Methods). Over 85 iterations of the LOOCV, the mean AUC was 0.98 (95\% confidence interval = 0.96-0.99), with a maximal specificity and sensitivity of 0.93 and 0.93 , respectively

also be used in the context of T cell-replete HSCT (that is, without the application of in vivo alemtuzumab). We identified an independent sample cohort of 32 HLAidentical HSCT donor-recipient sibling pairs. As before, patients were selected based on transplant outcomes to obtain evenly numbered sample groups, that is, severe aGVHD (grades III + IV; $\mathrm{n}=9$ ), mild aGVHD (grades I + II; $n=8$ ), and no aGVHD (grade 0; $n=15$ ). Further details about sample selection and characteristics of HSCT donor-recipient sibling pairs are provided in the Methods section and Table 1, respectively.

In agreement with the data obtained in donors in the context of $\mathrm{T}$ cell-depleted HSCT, we confirmed the DNA hypomethylation phenotype at the top-ranked DMP cg20475486 $(P=0.050$, Wilcoxon rank-sum test; Fig. 3 c). The area under the ROC curve was 0.73 at the DNA methylation threshold with the maximal specificity and sensitivity (Fig. 3d). In summary, we validated the top-ranked DMP associated with aGVHD severity status in donors in relation to both $\mathrm{T}$ cell-depleted and $\mathrm{T}$ cellreplete conditioning regimens for HSCT. Our findings describe the first epigenetic classifier for the identification of donors with an intrinsically increased alloresponse prior to HSCT, identifying donor grafts more appropriate to undergo $\mathrm{T}$ cell depletion to reduce aGVHD incidence.

\section{Biological significance of DMR associated with aGVHD severity on chromosome $14 \mathrm{q} 24.2$}

The DMR harboring the four top-ranked CpG classifiers (Fig. 2a) map to a CpG island at an intergenic region on chromosome 14q24.2. To investigate the potential functional role of this DMR, we annotated the genomic locus using available epigenomic reference datasets provided by the NIH Roadmap Epigenomics Project [36]. Specifically, we examined chromatin state maps of 22 primary hematopoietic cell types. Chromatin states are defined as spatially coherent and biologically meaningful combinations of distinct chromatin marks. These are systematically computed by exploiting the correlation of such marks, e.g., histone modifications, DNA methylation, and chromatin accessibility [37, 38]. This approach has recently been extended to include prediction (or 'imputation') of additional chromatin marks [39].

The annotation with both primary and imputed chromatin state maps revealed that the DMR is located at an active transcription start site or poised promoter in G-CSF-mobilized $\mathrm{CD}_{4} 4^{+}$hematopoietic stem cells, and a Polycomb-repressed region in $\mathrm{CD}^{+}$ $\mathrm{T}$ cells of peripheral blood (Additional file 4). The closest annotated gene is SMOC1 (SPARC related modular calcium binding 1), located $3.77 \mathrm{~kb}$ upstream of the top-ranked DMP cg20475486 (Table 2). 


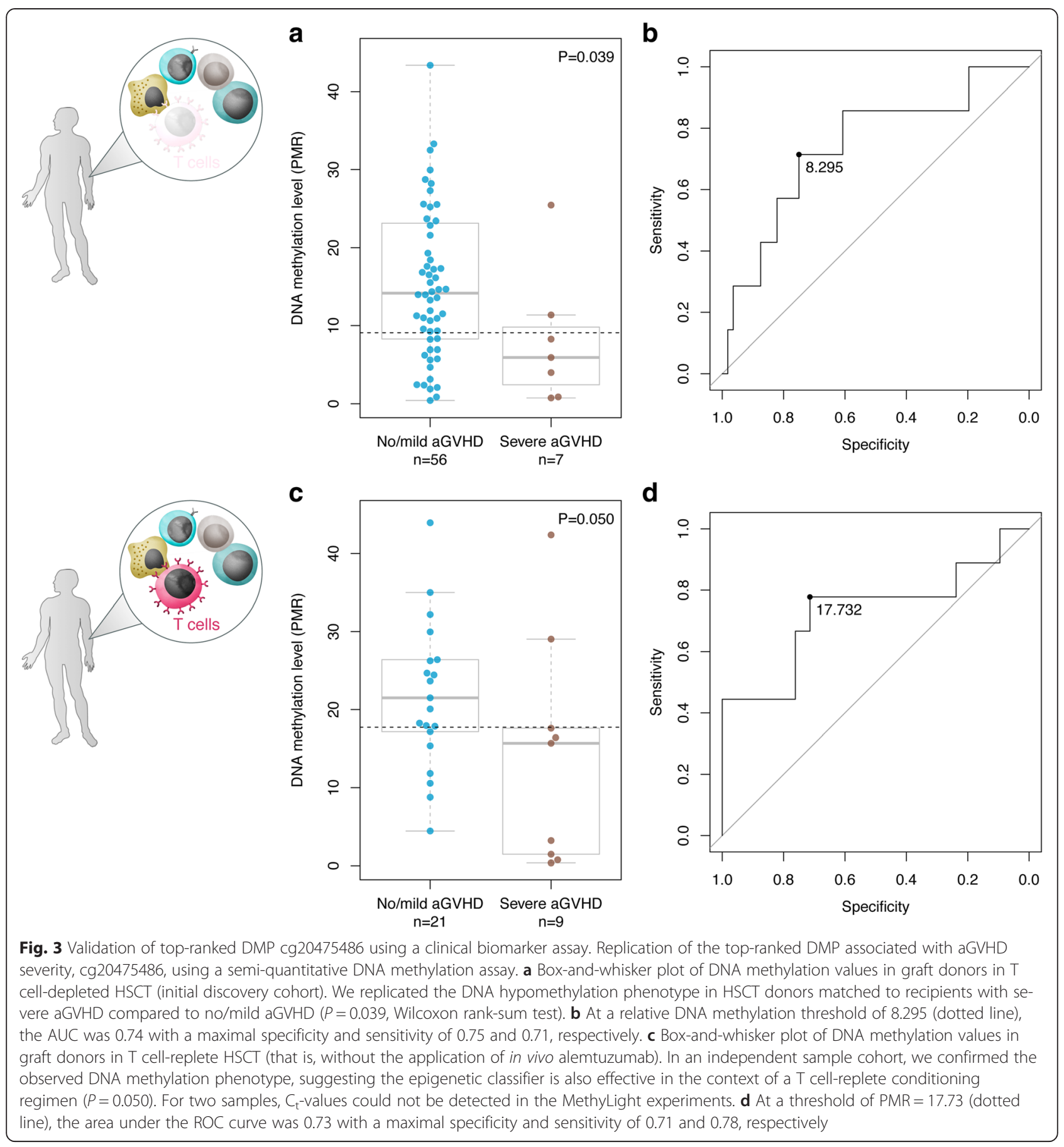

SMOC1 does not have a previously reported function in inflammatory or immune response pathways, and the evidence provided by the chromatin state maps suggests that SMOC1 is unlikely to be the relevant target gene at this locus. Instead, the DMR may pinpoint a transcription start site of a novel, unannotated gene or transcript that potentially plays a role in $\mathrm{T}$ cell lineage development.

\section{Discussion}

GVHD is a condition in which both prevention and treatment are associated with significant costs and morbidities. In this study, we derived the first HSCT donorspecific DNA methylation signature that predicts the incidence of severe aGVHD in HLA-matched sibling recipients. Following a genome-wide survey in 85 HSCT donors using $450 \mathrm{~K}$ arrays, we replicated the identified 
epigenetic signature associated with aGVHD severity status in 63 donors using MethyLight, a low-cost assay that is applicable for routine clinical diagnostics. Furthermore, we demonstrated the utility of the epigenetic classifier in the context of a $\mathrm{T}$ cell-replete conditioning regimen in an additional 32 HSCT donors.

We note that our study has limitations. Our DNA methylation analysis was carried out in peripheral blood, a substantially heterogeneous tissue. Cellular heterogeneity is a potential confounder in differential DNA methylation analyses [14, 40]. While we carefully assessed and controlled for differential leukocyte composition using statistical methods (Methods; Additional file 5), we cannot exclude the possibility that some of the identified DMPs are due to differential counts of cellular subpopulations that are not accounted for by the statistical inference. Indeed, previous studies have shown that most of the potential alloreactivity of a donor graft resides within the naive $\mathrm{T}$ cell pool [41]. Therefore, differences in cellular composition of a subset of alloreactive $\mathrm{T}$ cells may even be anticipated. However, it should be noted that even if the associations are observed as a result of differential cell composition, this does not affect the validity of our finding as a valuable classifier.

The discriminatory performance of the presented epigenetic classifier, which consisted of only the CpG classifier cg20475486 at the replication stage, was reduced compared to the classifier panel consisting of multiple $\mathrm{CpG}$ sites at the discovery stage. We investigated whether variation in distinct HSCT donor groups (that is, donors matched to recipients with no complications, and those matched to mild aGVHD) caused the reduced performance, but could not substantiate this hypothesis (Additional file 6). Instead, the reason could be technical, because the $450 \mathrm{~K}$ array platform measured DNA methylation levels at cg20475486 in single-nucleotide resolution, whereas MethyLight assessed the levels across eight linked CpGs (Additional file 1). Based on the combined graft donor pool across both $\mathrm{T}$ celldepleted and T cell-replete HSCT ( $\mathrm{n}=93$ donors; PMR $=8.295$ ), the AUC was 0.69 with a maximal specificity and sensitivity of 0.81 and 0.56 , respectively. The findings of our study will need validating in larger cohorts of HSCT donors that are matched to recipients with severe aGVHD. Also, we acknowledge that additional CpG classifiers are required to allow for effective routine clinical testing of graft donors prior to HSCT. Such additional CpG sites can be collated to constitute a more potent classifier panel, for example by drawing from the list of identified DMPs (Table 2). This strategy has previously been applied in an epigenetic biomarker panel for renal cell carcinoma [42] and active ovarian cancer [43] using 20 and even 2,714 distinct CpG classifiers, respectively. In addition, an independent discovery stage for HSCT donors whose recipients undergo T cell-replete conditioning may reveal a different set of DMPs.

The 450K array platform used for DNA methylation profiling holds a fixed, predesigned content covering less than $2 \%$ of all annotated CpG sites. It is conceivable that $\mathrm{CpG}$ sites that are not captured by the array are more informative. Our study design also required two sample batches, which necessitated batch effect correction, potentially reducing the number of informative DMPs (Methods). Nonetheless, if combined with an alternative assay for replication of initial discoveries, $450 \mathrm{~K}$ arrays are the current assay of choice for genomewide surveys due to their quantitative, robust, and scalable assessment of DNA methylation levels.

We recognize that the presented epigenetic changes associated with aGVHD severity status may in fact merely mediate genetic risk factors for HSCT. We omitted array probes from statistical analyses that contained common genetic variants that likely influence DNA binding (Methods), but DNA methylation levels may be mediated by genetic variants in proximity, that is, represent DNA methylation quantitative trait loci (metQTLs). Systematic met-QTL mapping efforts in HSCT donors with matched genotypic and epigenotypic datasets, combined with causal inference methods [14], are necessary to investigate this possibility further, but are beyond the scope of this study.

We provide evidence that DNA methylation signatures in graft donors associated with aGVHD severity in recipients correlate with well-characterized gene sets and molecular processes relevant to GVHD etiology, such as MHC class II restriction (Additional file 3). However, the functional importance of the specific DMR containing the four top-ranked $\mathrm{CpG}$ classifiers on chromosome 14q24.2 (Fig. 2a) is obscure and warrants additional experimental investigation. Annotation using chromatin state maps showed that the DMR maps to an active transcription start site in $\mathrm{CD}_{3} 4^{+}$hematopoietic stem cells, and a Polycomb-repressed regulatory element in $\mathrm{CD}^{+} \mathrm{T}$ cells (Additional file 4). Indeed, Polycomb proteins play a role in preventing the inappropriate hyperactivation of $\mathrm{T}$ cells in the setting of GVHD [44]. Future studies should delineate the DNA methylation signature in homogeneous $\mathrm{T}$ cell subsets and at various stages of $\mathrm{T}$ cell formation and development. International consortia, in particular BLUEPRINT [45], add further reference epigenomes of hematopoietic cell types, including many progenitor populations.

\section{Conclusions}

Our results are the first to identify an epigenetic signature in healthy graft donors that can predict aGVHD in recipients. The findings suggest the possible use of epigenetic profiling in conjugation with genetic profiling to 
improve donor selection prior to HSCT and inform immunosuppressive transplant conditioning, with the paramount goal of improving patient outcomes. Looking ahead, we plan to further develop this first epigenetic classifier and its utility to also include unrelated HSCT donors, who constitute the majority of the allogeneic HSCT donor pool, and for which the incidence of aGVHD is most prevalent.

\section{Additional files}

Additional file 1: PCR primers and probes used in MethyLight replication experiments. A total of three MethyLight reactions were designed, which targeted top-ranked DMPs associated with aGVHD severity: cg10399005, cg20475486, and cg07280807. Of these reactions, cg20475486 achieved the highest PCR efficiency and was subsequently used in validation experiments (highlighted in gray). Start and end positions of primer and probes are noted in relation to the design start coordinates. Chromosomal positions are reported in genome build $=$ hg19. (PDF $110 \mathrm{~kb}$ )

Additional file 2: Quality assessment of the Illumina Infinium HumanMethylation 450 assay. The quality of the $450 \mathrm{~K}$ array data was assessed after normalization, probe filtering, and batch correction (for details see Methods). (a) Density of DNA methylation $\beta$-values. (b) Multidimensional scaling (MDS) indicates the similarities and differences of samples by calculating the Euclidean distances between samples based on all CpG sites, and then projecting these distances into 2D coordinates. We found Dimension 4 to associate with aGVHD severity, accounting for $3 \%$ of the total variance. HSCT donors matched to healthy recipients and those matched to recipients diagnosed with mild aGVHD could not be stratified using MDS. Therefore, these two sample groups were combined for subsequent analysis. (c) Singular value decomposition (SVD) determines the nature of the largest components of variation (Teschendorff AE et al. PLoS One. 2009;4:e8274). We assessed the first six principal components (PCs), and correlated these to phenotypic factors of donors (e.g., sex, age at transplant, and CMV serostatus), phenotypic factors of recipients (e.g.t aGVHD status and severity), factors related to the experimental setup (e.g., Sentrix ID, sample plate, and sample well), as well as internal control parameters (e.g., bisulfite conversion efficiency). DNA methylation age ('DNAm age') was predicted based on the raw DNA methylation data using the DNA Methylation Age Calculator (https://dnamage. genetics.ucla.edu/), as described by Horvath (Horvath S. Genome Biol. 2013;14:R115). The phenotypic factor 'transplant date' denotes the year of the day of the graft transplant (day 0). We found PC4 and PC5 to most strongly correlate with aGVHD severity, achieving a significance level of $P<0.01$ and $P<1 \times 10^{-5}$, respectively. (PDF $507 \mathrm{~kb}$ )

Additional file 3: Ontology annotation of genes flanking DMRs using GREAT. We report the ontology of genes flanking the 453 identified DMRs. Specifically, we indicate all enriched terms in the binomial test over genomic regions at an FDR of less than $5 \%$. (PDF $107 \mathrm{~kb}$ )

Additional file 4: Annotation of top-ranked DMPs using epigenomic reference datasets. The genomic locus on chromosome $14 \mathrm{q} 24.2$ (position $=70,261,006-70,349,114$; genome build $=$ hg19) harboring the top-ranked DMR is shown using the WashU Epigenome Browser v40.0.0 (http://epigenomegateway.wustl.edu/browser/). The top-ranked DMR containing CpG classifiers of aGVHD severity (Table 2) is located at a CpG island (position =70,316,847-70,317,240; indicated with an orange arrow). RefSeq and Gencode v17 genes, as well as CpG islands, are shown in the bottom panel of the figure. A total of 50 epigenomic reference tracks provided by the NIH Roadmap Epigenomics Project are displayed. Specifically, we show both the primary and imputed chromatin state maps in 22 primary hematopoietic cell types. The highlighted DMR overlaps with an active transcription start site (red) or poised promoter (pink) in G-CSF-mobilized CD34+ hematopoietic stem cells, and a Polycombrepressed region in $\mathrm{CD}^{+} \mathrm{T}$ cells of peripheral blood. (PDF $960 \mathrm{~kb}$ )
Additional file 5: Estimation of differential leukocyte counts. For each sample, the composition of major leukocyte cell types was estimated using DNA methylation signatures of an external reference set consisting of purified leukocytes (Houseman EA et al. BMC Bioinformatics. 2012;13:86). The leukocyte composition was grouped for each measured cell type, and stratified for donors paired with healthy recipients $(n=39)$, recipients developing mild $(n=37)$ and severe aGVHD $(n=9)$. We did not observe significant differences $(P<0.05)$ in cellular composition between the sample groups. For each cell type, the bar indicates the median of the composition estimate. Error bars indicate $95 \%$ confidence intervals. $P$ values were calculated using a Kruskal-Wallis rank-sum test. (PDF $235 \mathrm{~kb}$ )

Additional file 6: Box-and-whisker plots of DNA methylation values in graft donors in the discovery cohort assessed using MethyLight and $450 \mathrm{~K}$ arrays. Only HSCT donors of the discovery cohort that were profiled on both assay platforms are shown. We identified a DNA hypomethylation phenotype at the top-ranked DMP cg20475486 in graft donors matched to recipients with severe aGVHD. HSCT donors matched to healthy recipients and those matched to recipients diagnosed with mild aGVHD could not be discriminated. (PDF $256 \mathrm{~kb}$ )

\section{Abbreviations}

aGVHD: acute graft-versus-host disease; AUC: area under the curve; CGI: CpG island; DMP: differentially methylated position; DMR: differentially methylated region; DNAm: DNA methylation; FDR: false-discovery rate; G-CSF: granulocyte-colony stimulating factor; HLA: human leukocyte antigen; HSCT: hematopoietic stem cell transplantation; IGR: intergenic region; LOOCV: leave-one-out cross-validation; MDS: multidimensional scaling; MHC: major histocompatibility complex; PMR: percentage of methylated reference; ROC: receiver operating characteristic; TSS200/TSS1500: within 200/ $1,500 \mathrm{bp}$ of a transcription start site.

\section{Competing interests}

The authors declare that they have no competing interests.

\section{Authors' contributions}

DSP, VKR, KSP, and SB designed the study. DSP and AJ performed 450K array and MethyLight experiments, respectively. DSP analyzed all data and performed statistical analyses. AF helped with statistical analyses and interpretation of results. APW prepared ethics application. RSS, NPM, NA, RS, and RMS provided clinical samples and phenotype information. JFA, MW, SM, SGEM, JAM, VKR, KSP, and SB supervised the study. DSP wrote the manuscript. All authors read and approved the final manuscript.

\section{Acknowledgments}

We would like to thank Christoph Bock (CeMM Research Center for Molecular Medicine), James Barrett, Lee Butcher, and Miljana Tanic (UCL Cancer Institute) for advice on statistical analyses; Kerra Pearce and Mark Kristiansen (UCL Genomics) for processing the Illumina Infinium HumanMethylation450 BeadChips; and Laura Phipps for proofreading the manuscript. This work was funded by the EU-FP7 Project BLUEPRINT (282510), Barts Charity (838/2030), Anthony Nolan, and Wellcome Trust (99148). We acknowledge support from a CRUK Clinical Research Fellowship (RSS). The research was funded in part by the National Institute for Health Research (NIHR) Blood and Transplant Research Unit (BTRU) in Stem Cells and Immunotherapy at UCL in partnership with NHS Blood and Transplant (NHSBT), and in collaboration with UCL Hospitals and Imperial College Healthcare NHS Trust, which received support from the Department of Health and CRUK funding schemes for NIHR Biomedical Research Centres and Experimental Cancer Medicine Centres.

\section{Author details}

'UCL Cancer Institute, University College London, London, UK. ${ }^{2}$ Department of Women's Cancer, UCL Elizabeth Garrett Anderson Institute for Women's Health, University College London, London, UK. ${ }^{3}$ Department of Haematology, University College London, University College London Hospital, London, UK. ${ }^{4}$ Anthony Nolan Research Institute, Royal Free Hospital, London, UK. ${ }^{5}$ Department of Haematology, University College London, Royal Free Hospital, London, UK. ${ }^{6}$ Clinical Immunology, Imperial NHS Trust Hammersmith Hospital, London, UK. ${ }^{7}$ Centre for Haematology, Faculty of Medicine, Imperial College London, Hammersmith Hospital, London, UK. 
${ }^{8}$ Department of Clinical Haematology, Imperial College Healthcare NHS Trust, Hammersmith Hospital, London, UK. ${ }^{9}$ Blizard Institute, Barts and The London School of Medicine and Dentistry, Queen Mary University of London, London, UK.

Received: 26 July 2015 Accepted: 12 November 2015 Published online: 15 December 2015

\section{References}

1. McDonald-Hyman C, Turka LA, Blazar BR. Advances and challenges in immunotherapy for solid organ and hematopoietic stem cell transplantation. Sci Transl Med. 2015;7:280rv282.

2. Holtan SG, Pasquini M, Weisdorf DJ. Acute graft-versus-host disease: a bench-to-bedside update. Blood. 2014;124:363-73.

3. Welniak LA, Blazar BR, Murphy WJ. Immunobiology of allogeneic hematopoietic stem cell transplantation. Annu Rev Immunol. 2007;25:139-70.

4. Ho VT, Soiffer RJ. The history and future of T-cell depletion as graft-versushost disease prophylaxis for allogeneic hematopoietic stem cell transplantation. Blood. 2001:98:3192-204.

5. Gooley TA, Chien JW, Pergam SA, Hingorani S, Sorror ML, Boeckh M, et al. Reduced mortality after allogeneic hematopoietic-cell transplantation. N Engl J Med. 2010;363:2091-101.

6. Levine JE, Logan BR, Wu J, Alousi AM, Bolaños-Meade J, Ferrara JLM, et al. Acute graft-versus-host disease biomarkers measured during therapy can predict treatment outcomes: a Blood and Marrow Transplant Clinical Trials Network study. Blood. 2012:119:3854-60.

7. Vander Lugt MT, Braun TM, Hanash S, Ritz J, Ho VT, Antin JH, et al. ST2 as a marker for risk of therapy-resistant graft-versus-host disease and death. N Engl J Med. 2013;369:529-39.

8. Cedar H, Bergman Y. Epigenetics of haematopoietic cell development. Nat Rev Immunol. 2011;11:478-88.

9. Wei G, Wei L, Zhu J, Zang C, Hu-Li J, Yao Z, et al. Global mapping of H3K4me3 and H3K27me3 reveals specificity and plasticity in lineage fate determination of differentiating CD4+ T cells. Immunity. 2009;30:155-67.

10. Ohkura N, Hamaguchi M, Morikawa H, Sugimura K, Tanaka A, Ito $Y$, et al. $T$ cell receptor stimulation-induced epigenetic changes and Foxp3 expression are independent and complementary events required for Treg cell development. Immunity. 2012;37:785-99.

11. Allan RS, Zueva E, Cammas F, Schreiber HA, Masson V, Belz GT, et al. An epigenetic silencing pathway controlling $T$ helper 2 cell lineage commitment. Nature. 2012;487:249-53.

12. Rakyan VK, Beyan H, Down TA, Hawa MI, Maslau S, Aden D, et al. Identification of type 1 diabetes-associated DNA methylation variable positions that precede disease diagnosis. PLoS Genet. 2011;7:e1002300.

13. Absher DM, Li X, Waite LL, Gibson A, Roberts K, Edberg J, et al. Genomewide DNA methylation analysis of systemic lupus erythematosus reveals persistent hypomethylation of interferon genes and compositional changes to CD4+ T-cell populations. PLoS Genet. 2013;9:e1003678.

14. Liu Y, Aryee MJ, Padyukov L, Fallin MD, Hesselberg E, Runarsson A, et al. Epigenome-wide association data implicate DNA methylation as an intermediary of genetic risk in rheumatoid arthritis. Nat Biotechnol. 2013;31:142-7.

15. Rodriguez RM, Suarez-Alvarez B, Salvanés R, Muro M, Martínez-Camblor P, Colado $E$, et al. DNA methylation dynamics in blood after hematopoietic cell transplant. PLoS One. 2013;8:e56931.

16. Timp W, Feinberg AP. Cancer as a dysregulated epigenome allowing cellular growth advantage at the expense of the host. Nat Rev Cancer. 2013;13:497-510.

17. Peggs KS, Hunter A, Chopra R, Parker A, Mahendra P, Milligan D, et al. Clinical evidence of a graft-versus-Hodgkin's-lymphoma effect after reduced-intensity allogeneic transplantation. Lancet. 2005;365:1934-41.

18. Glucksberg H, Storb R, Fefer A, Buckner CD, Neiman PE, Clift RA, et al. Clinical manifestations of graft-versus-host disease in human recipients of marrow from HL-A-matched sibling donors. Transplantation. 1974;18:295-304.

19. Fortin JP, Labbe A, Lemire M, Zanke BW, Hudson TJ, Fertig EJ, et al. Functional normalization of 450K methylation array data improves replication in large cancer studies. Genome Biol. 2014;15:503.

20. Aryee MJ, Jaffe AE, Corrada-Bravo H, Ladd-Acosta C, Feinberg AP, Hansen KD, et al. Minfi: a flexible and comprehensive Bioconductor package for the analysis of Infinium DNA methylation microarrays. Bioinformatics. 2014;30:1363-9.
21. Triche Jr TJ, Weisenberger DJ, Van Den Berg D, Laird PW, Siegmund KD. Low-level processing of Illumina Infinium DNA Methylation BeadArrays. Nucl Acids Res. 2013;41:E90.

22. Grundberg E, Meduri E, Sandling JK, Hedman ÅK, Keildson S, Buil A, et al. Global analysis of DNA methylation variation in adipose tissue from twins reveals links to disease-associated variants in distal regulatory elements. Am J Hum Genet. 2013;93:876-90.

23. Johnson WE, Li C. Adjusting batch effects in microarray expression data using empirical Bayes methods. Biostatistics. 2007;8:118-27.

24. Leek JT, Johnson WE, Parker HS, Jaffe AE, Storey JD. The sva package for removing batch effects and other unwanted variation in high-throughput experiments. Bioinformatics. 2012;28:882-3.

25. Houseman EA, Accomando WP, Koestler DC, Christensen BC, Marsit CJ, Nelson $\mathrm{HH}$, et al. DNA methylation arrays as surrogate measures of cell mixture distribution. BMC Bioinformatics. 2012;13:86.

26. Butcher LM, Beck S. Probe Lasso: a novel method to rope in differentially methylated regions with 450K DNA methylation data. Methods. 2015;72:21-8.

27. Smyth GK. Linear models and empirical bayes methods for assessing differential expression in microarray experiments. Stat Appl Genet Mol Biol. 2004;3:Article 3.

28. McLean CY, Bristor D, Hiller M, Clarke SL, Schaar BT, Lowe CB, et al. GREAT improves functional interpretation of cis-regulatory regions. Nat Biotechnol. 2010;28:495-501.

29. Tibshirani R, Hastie T, Narasimhan B, Chu G. Diagnosis of multiple cancer types by shrunken centroids of gene expression. Proc Natl Acad Sci U S A. 2002;99:6567-72.

30. Tibshirani R, Hastie T, Narasimhan B, Chu G. Class prediction by nearest shrunken centroids, with applications to DNA microarrays. Stat Sci. 2003;18: 104-17.

31. Robin X, Turck N, Hainard A, Tiberti N, Lisacek F, Sanchez JC, et al. pROC: an open-source package for $\mathrm{R}$ and $\mathrm{S}+$ to analyze and compare ROC curves. BMC Bioinformatics. 2011;12:77.

32. Bibikova M, Barnes B, Tsan C, Ho V, Klotzle B, Le JM, et al. High density DNA methylation array with single CpG site resolution. Genomics. 2011;98:288-95.

33. Eads CA, Danenberg KD, Kawakami K, Saltz LB, Blake C, Shibata D, et al. MethyLight: a high-throughput assay to measure DNA methylation. Nucl Acids Res. 2000;28:E32.

34. Payne SR. From discovery to the clinic: the novel DNA methylation biomarker (m)SEPT9 for the detection of colorectal cancer in blood. Epigenomics. 2010;2:575-85.

35. Mikeska T, Bock C, Do H, Dobrovic A. DNA methylation biomarkers in cancer: progress towards clinical implementation. Expert Rev Mol Diagn. 2012:12:473-87.

36. Roadmap Epigenomics Consortium. Integrative analysis of 111 reference human epigenomes. Nature. 2015;518:317-30.

37. Ernst J, Kellis M. Discovery and characterization of chromatin states for systematic annotation of the human genome. Nat Biotechnol. 2010;28:817-25.

38. Ernst J, Kellis M. ChromHMM: automating chromatin-state discovery and characterization. Nat Methods. 2012;9:215-6.

39. Ernst J, Kellis M. Large-scale imputation of epigenomic datasets for systematic annotation of diverse human tissues. Nat Biotechnol. 2015;33:364-76.

40. Paul DS, Beck S. Advances in epigenome-wide association studies for common diseases. Trends Mol Med. 2014;20:541-3.

41. Ghosh A, Holland AM, van den Brink MRM. Genetically engineered donor $T$ cells to optimize graft-versus-tumor effects across MHC barriers. Immunol Rev. 2014;257:226-36.

42. Lasseigne BN, Burwell TC, Patil MA, Absher DM, Brooks JD, Myers RM. DNA methylation profiling reveals novel diagnostic biomarkers in renal cell carcinoma. BMC Medicine. 2014;12:235.

43. Teschendorff AE, Menon U, Gentry-Maharaj A, Ramus SJ, Gayther SA Apostolidou S, et al. An epigenetic signature in peripheral blood predicts active ovarian cancer. PLoS One. 2009;4:e8274.

44. Onodera A, Nakayama T. Epigenetics of T cells regulated by Polycomb/ Trithorax molecules. Trends Mol Med. 2015;21:330-40.

45. Adams D, Altucci L, Antonarakis SE, Ballesteros J, Beck S, Bird A, et al. BLUEPRINT to decode the epigenetic signature written in blood. Nat Biotechnol. 2012;30:224-6. 